\title{
Intelligent Transmitter of Field (ITF) Based on Microcontroller for Data Acquisition in PV Solar Plants
}

\author{
Herminio Martínez-García and Encarna García-Vílchez \\ Eastern Barcelona School of Engineering (Escuela de Ingeniería de Barcelona Este - EEBE) \\ Department of Electronics Engineering \\ Technical University of Catalonia (UPC). BarcelonaTech \\ Diagonal-Besòs Campus. Eduard Maristany Ave., nº 10 - 14 \\ 08019 - Barcelona. SPAIN \\ Phone Number: +34.93.413.72.90; Fax Number: +34.93.413.74.01 \\ E-mail: herminio.martinez@upc.edu
}

\begin{abstract}
In this article the design of a prototype of an Intelligent Transmitter of Field (ITF) is presented. The objective of this ITF is to be used as a data acquisition in a PV solar plant implemented at the new Campus, the Diagonal-Besòs Campus, of the Technical University of Catalonia - BarcelonaTech (UPC). Aspects like power consumption of the electronic circuits, energy supply through the wires of communication of the transmitter, the coexistence in these wires of analogical signals of measurement and digital signals of communication, etc., are essential to get the foreseen objective.
\end{abstract}

The article seeks, also, to contribute to obtain a practical and, at the same time, didactic solution for a problem that has been one of the main challenges in instrumentation field for data acquisition in renewable energy systems.

\section{Key words}

Data Acquisition, Microcontroller-Based Systems, Industrial Communications, Digital Communications, Renewable Energies.

\section{Introduction}

Certain applications demand the use of transmitters whose energy supply is carried out through the own line for which they send the signal measured in the receiver. This is the case of data acquisition subsystems in order to monitor significant variables in renewable energy subsystems.

In places of difficult access, such as PV solar plants, it is convenient, also, that the confirmations and periodic calibrations of the transmitter are carried out in a comfortable way [1]. This will be possible if the transmitter is endowed with the capacity of "to dialogue" with the operator without altering the measure signal that is transmitting. The design and development of any new transmitter takes implicit the necessity to be compatible with the analogical conventional transmitters and, also, to enlarge its operative benefits. Therefore, a habitual demand is the ability to substitute the conventional transmitters without the necessity of a new wiring or to alter the existent ones [1].

In this work the study, design and implementation of an Intelligent Transmitter of Field, (ITF), is presented. The ITF has to be able to take the supply energy and to establish the digital communication with the receiver only through two wires. At the same time, it is required to maintain the compatibility with the analogical traditional transmitters of 4 ... $20 \mathrm{~mA}$. This aspect will allow that [2], besides carrying out the classical functions of its predecessors, these transmitters can be part as active elements in a more complex distributed control system (Fig. 1).

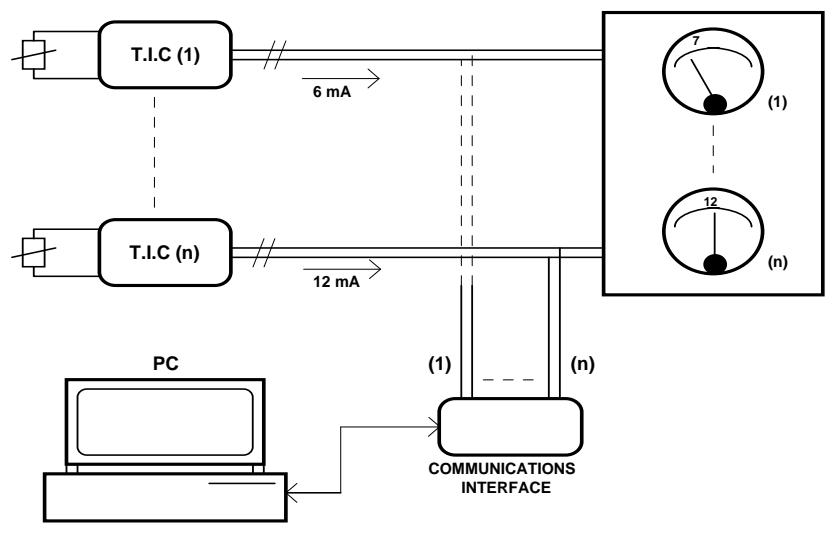

Fig. 1. Distributed data acquisition system.

\section{Conception Engineering and Design System}

The above features for an ITF define certain characteristics that the transmitter should include in its design phase. From the functional point of view, it required that: 
a) The measure signal, in current form in the range from 4 to $20 \mathrm{~mA}$, should be exclusively function of the physical variable measured by the sensor. This signal will not be altered by the intensity consumed by the electronics of the transmitter.

b) In normal operation (transmitter connected to the receiver and the whole operative group), the signal of digital communication should have the characteristic of not perturbing the measure signal sent by the transmitter. In this way, the transmitter will be provided with functions such as: calibration, periodic confirmation, modification of the measure range, etc.

These previous requirements lead to solve the following technical aspects:

a) Reduced consumption of the transmitter electronics for not influencing significantly in the magnitude of the measure current.

b) Communication technique that presents the previously mentioned characteristics and that it doesn't alter the balance of present currents in the transmitter.

To give an answer to the outlined questions, we present the alternative design shown in Fig. 2. In this figure we observe the inclusion of a current generator governed by voltage (G1) whose intensity in the range from 2 to $18 \mathrm{~mA}$ will be exclusively function of the signal provided by the sensor. If we supply energy to the whole electronics by means of another generator (G2) with constant current of 2 $m A$, we will have in the communication line an analogical signal with a current inside the range of $4 \ldots 20 \mathrm{~mA}$ whose variation will only be function of the physical magnitude measurement.

The internal electronics would be made up basically by the input bridge, a PGDA (programmable gain differential amplifier), the converter $\mathrm{A} / \mathrm{D}, \mathrm{D} / \mathrm{A}$, and the microcontroller, $\mu \mathrm{C}$. The communication signal, of digital nature, would be coupled to the line through capacitor $C_{1}$.

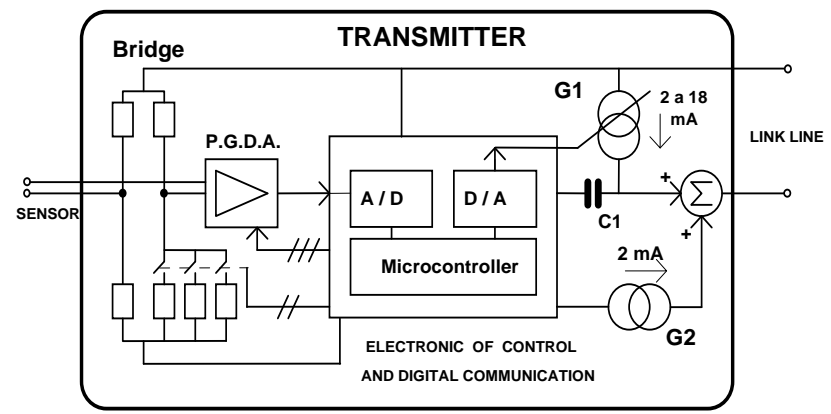

Fig. 2. Block Diagram of the ITF.

\section{A. Input Bridge}

The conditioning electronics for the signal coming from the sensor is implemented by a Wheatstone bridge allowing inputs coming from thermocouples, signals in current of $4 \ldots 20 \mathrm{~mA}$, resistances-temperature Pt-100 $\Omega$ or voltages in the range of $0 \ldots 100 \mathrm{mV}$ and $0 \ldots 1 \mathrm{~V}$. This electronic circuit will be protected with input varistors and with a circuit against the break of the thermocouple.

\section{B. Amplifier Circuit}

The amplifier will be made up by a PGDA (INA118). Depending on the chosen sensor, the amplifier will generate the corresponding output signal with the appropriate gain. This component presents a reduced consumption, a great linearity and a low thermal bias [3].

\section{Analog-to-Digital Conversion Circuit}

This block contains the 12-bit LTC1288 converter (Fig. 3). It has two input channels: The first is used as main channel of measurement, and the second is useful for sensing the environment temperature of the transmitter (this is necessary to compensate thermocouple signals), and for sensing the precision voltage reference (useful in the compensation of temporary drift).

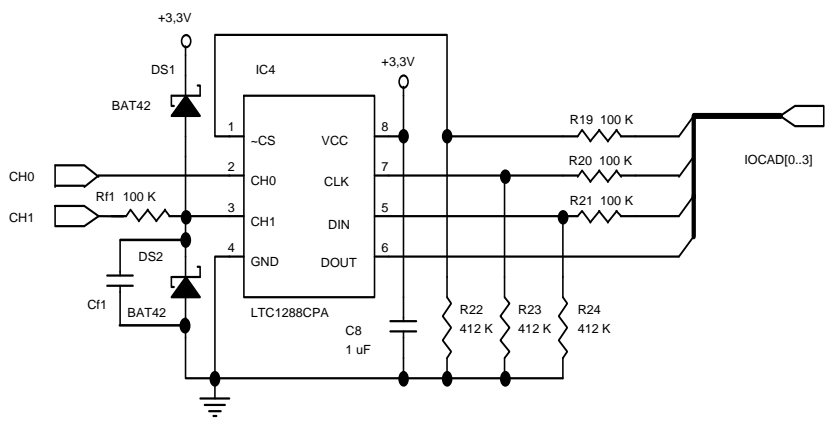

Fig. 3. Analog-to-digital conversion circuit.

\section{Microcontroller Circuit}

Based on a simple $\mu \mathrm{C}$, the PIC16C65 model [4], this circuit operates at a low clock frequency to reduce power consumption. The $\mu \mathrm{C}$ will acquire the measurement, it will perform a linearization and finally it will control the DAC. Furthermore, the microcontroller will be responsible for interpreting messages received via the line that connects the receiver (Fig. 4).

\section{E. Digital-to-Analog Conversion Circuit (DAC)}

This block includes the digital-analogical converter MAX 538 [5], serial type, with 12-bit resolution. It presents excellent characteristics of consumption (Fig. 5).

\section{F. Analog Output Circuit}

This circuit has the task of collecting the analogical signal provided by the DAC and 'adjust' it within the range of $4 \ldots$ $20 \mathrm{~mA}$. The current flows through the line that connects the transmitter and measurement equipment. 
The most important element of this stage is the XTR-101 circuit. This is a device that changes its transconductance depending on the voltage signal applied to its input. It has an auxiliary power supply, $2 m A$, which will create the whole power system. This value of current $(2 \mathrm{~mA})$ is the maximum allowed value for the consumption of all the electronic circuits that the transmitter incorporates (Fig. 6).

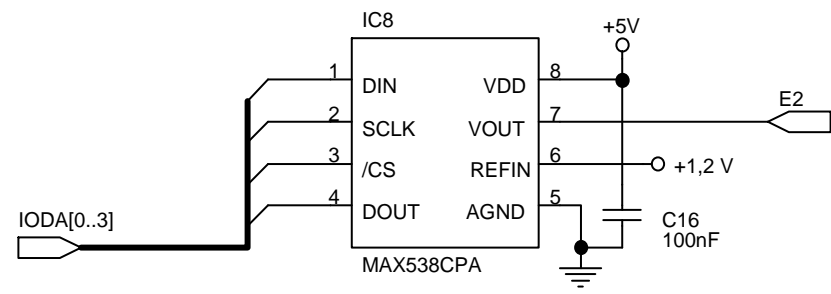

Fig. 5. Digital-analogical conversion circuit.

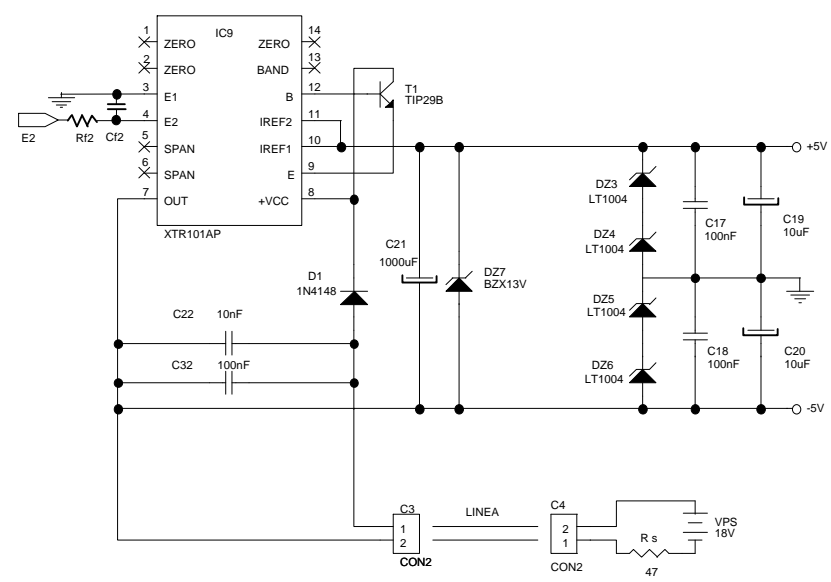

Fig. 6. Analog output circuit.

\section{G. Digital Communication System.}

An example of this type of communication in the transmission part is used in the instrumentation that uses HART protocol. As one of the objectives pursued in the design is teaching, we design a particular method of communication which modifies the intensity in the line of communication without changing its mean value (Fig. 7).

The digital communication will consist of two almost identical blocks, one located in the transmitter and the other located at the opposite end of the link line, i.e. close to the measuring equipment [6]. The latter constitutes a communication interface of a personal computer acting as the console.

The circuit of Fig. 7 is divided into three distinct parts:

a) On the left there is the module located in the transmitter and it is in turn divided into three blocks: The operational amplifier (block reception), the capacitor $C_{1}$ and $R_{8}$ (block transmission) and the generator $I_{1}$ flow that simulates the output of the XTR101.

b) In the central part there is the module that simulates the impedance of the line. For a length of $300 \mathrm{~m}$, it consists of $R_{L 1}=R_{L 2}=10 \Omega, L_{1}=L_{2}=300 \mu \mathrm{H}$ and, $C_{L}=50$ $n F$.

c) To the right there is the module located in the receiver and it is in turn divided into three blocks: The operational amplifier (block reception), the capacitor $C_{3}$ and $R_{9}$ (block transmission), the power line formed by the $18 \mathrm{~V}$ and the shunt resistance of 47 $\Omega$ (from which the signal of measurement is obtained).
TRANSMITTER

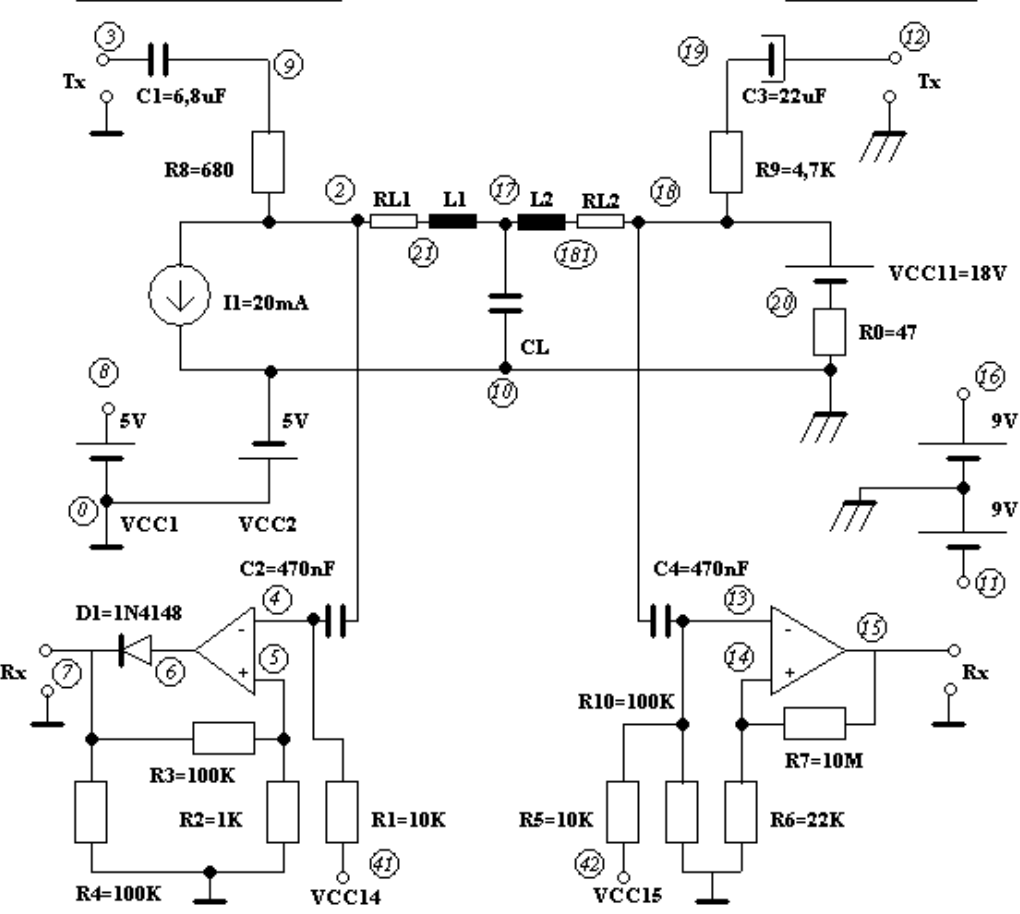

Fig. 7. Digital communication system. 


\section{Simulation Results}

Fig. 8 shows the chronograms of a digital communication system simulation (circuit in Fig. 7), in a communication from the transmitter to the receiver at $4800 \mathrm{bps}$ with a $300-$ meter twisted pair line.

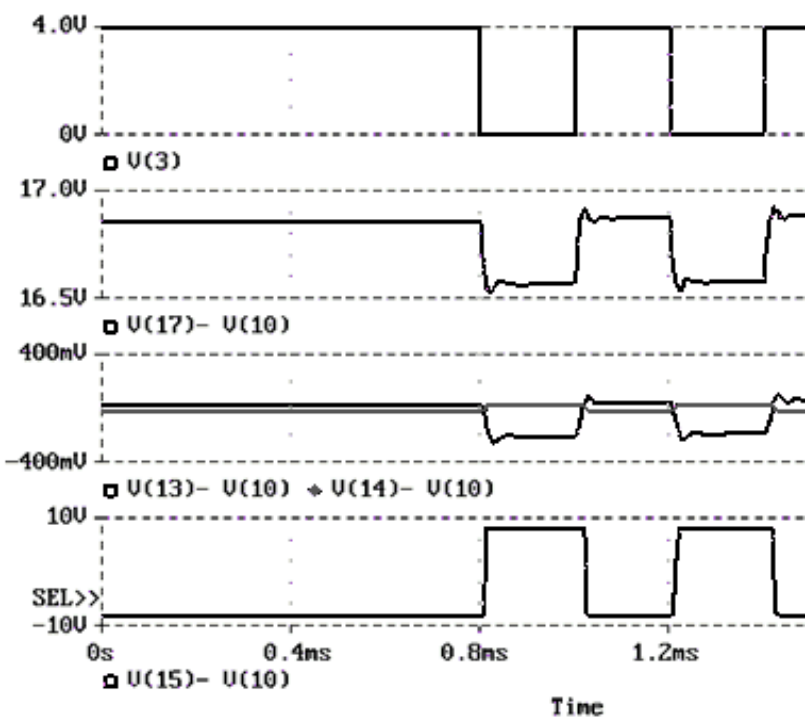

Fig. 8. Communication from transmitter to receiver with a 300 meter line.

Fig. 9 shows the chronograms of a digital communication system simulation (circuit in Fig. 7), in a communication from the receiver to the transmitter at $4800 \mathrm{bps}$ with a 300 meter twisted pair line.

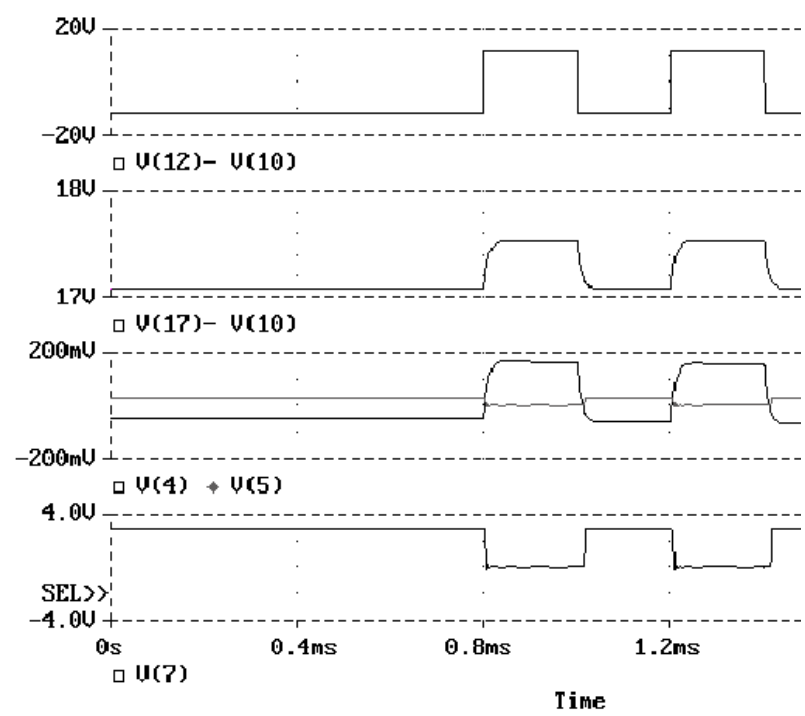

Fig. 9. Communication from receiver to transmitter with a 300 meter line.

The average value of the variation of intensity in the line, produced by the messages (asked/answered $V(17)-V(10)$ in Fig. 8 and Fig. 9) in a communication, must be equal to zero [7]. The signal $i(t)$ is the corresponding mixture of measurement signal $\left(I_{m}\right)$ and digital communication signal.
At every instant the integral of $i(t)$, between $t_{1}$ and $t_{2}$, is equal to $I_{m}$ (Fig. 10).

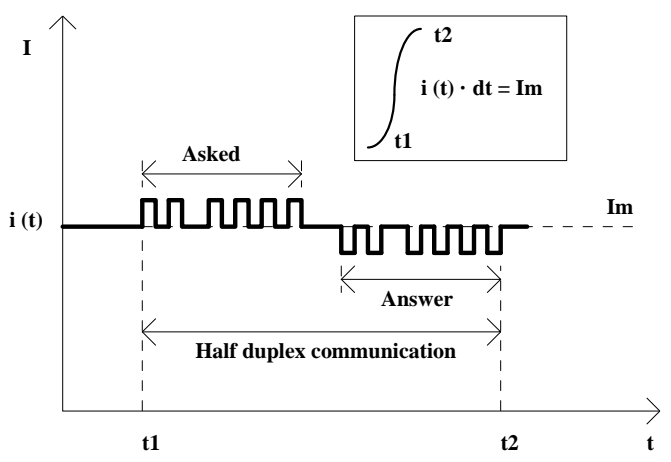

Fig. 10. Digital communication features.

\section{Conclusion}

The transmitter that has been designed and developed meets perfectly the original specifications in functionality and performance. This can be an important benchmark in the design and manufacturing of transmitters with a higher degree of sophistication, especially in the monitoring and data acquisition of significant variables in renewable energy subsystems.

Finally, Table I shows some of the most important characteristics of the transmitter.

\section{Acknowledgement}

This work has been partially supported and funded by the Spanish Ministerio de Economía y Competitividad by projects DPI2013-47799-C2-2-R, and DPI2016-78957-R, the LOGIMATIC EU project H2020-Galileo-2015687534 .

\section{References}

[1] Endress+Hauser, "Field Communication for Process Engineering”, 1995

[2] Ramakant, Sokoloff, "Analog and Digital Control Systems", Cambridge Press, 1998.

[3] Burr-Brown, "Linear Products". Burr-Brown, 1994

[4] MICROCHIP, "PIC 16/17 Microcontroller Data Book". Microchip, 1998.

[5] MAXIM, "New releases data book. Volume V'. Maxim, 2000.

[6] Texas Instruments, "Data Transmission Circuits". Texas Instruments, 1995.

[7] Bruce Carlson. "Communication systems", Mc Graw Hill, 2007.

Table I. - Designed ITF Features

\begin{tabular}{|l|}
\hline Signal of measurement: J, K, S, T, R, B, E, Pt-100, $100 \mathrm{mV}, 1 \mathrm{~V}$, and $0 / 4$ \\
$\ldots 20 \mathrm{~mA}$. \\
\hline Linearization within the response curves of thermocouples. \\
\hline Automatic cold junction compensation and correction of drift time. \\
\hline Software for the adjustment channels measurement. \\
\hline Software for changing scales and ranges of work. \\
\hline Communication: 4800 or 9600 baud \\
\hline Maximum distance between transmitter and receiver: 300 meters. \\
\hline Powered by the connection wires. \\
\hline Analog signal compatible with digital communication. \\
\hline
\end{tabular}


